\title{
AN OBSTRUCTION TO SMOOTH ISOTOPY IN DIMENSION 4
}

\section{DANIEL RUBERMAN}

\section{Introduction}

It is certainly well-known that a suitable count of solutions to the Yang-Mills or Seiberg-Witten equations gives rise to invariants of a smooth 4-manifold. In recent years, these invariants have become reasonably computable, and have led to many advances in 4-manifold theory. In this paper, we use the tools of gauge theory to describe invariants of a diffeomorphism $f$ which depend only on the isotopy class of $f$. The main application of this study is the construction of a diffeomorphism $f$ of a smooth 4-manifold which is homotopic to the identity, and the proof that it is not smoothly isotopic to the identity. It follows from work of Perron [17, 18] and Quinn [19, 8] (see also [13]) that $f$ is topologically isotopic to the identity.

Formally, the construction of gauge-theoretic invariants of a single space proceeds as follows. Under suitable topological hypotheses, the solution space to the Yang-Mills or Seiberg-Witten equations, denoted $\mathcal{M}(Y ; h)$, can be arranged to be compact and 0-dimensional. The parameter $h$ lives in some contractible space $\Pi$ which would be the space of metrics on $Y$ for Yang-Mills invariants, or the space $\left\{(g, \delta) \mid *_{g} \delta=\delta\right\} \subset \operatorname{Met}(Y) \times \Omega^{2}(Y)$ for the Seiberg-Witten invariants. One then defines $I(Y)$ to be \# $\mathcal{M}(Y ; h)$, where the symbol '\#' denotes an algebraic count of solutions. While the equations (and therefore their space of solutions!) involve the parameter $h$, the parameter space $\Pi$ is connected, so the algebraic count of solutions is independent of $h$. Hence $I(Y)$ depends only on the $C^{\infty}$ manifold $Y$.

The underlying idea for getting invariants of diffeomorphisms is similar; it occurs elsewhere in gauge theory [6] and in the context of symplectic geometry as well $[1,11,14,22]$. Suppose that for some manifold $Y$, the moduli space $\mathcal{M}(Y ; h)$ is formally of dimension -1 , and hence empty for generic $h \in \Pi$. If $f: Y \rightarrow Y$ is a diffeomorphism, then we can choose a generic path $h_{t}$ from $h_{0}=h$ to $h_{1}=f^{*}(h)$, and construct the 1-parameter family

$$
\tilde{\mathcal{M}}=\bigcup_{t} \mathcal{M}\left(Y ; h_{t}\right)
$$

One then defines an invariant $I(f)$ as the algebraic count of elements in this 0 -dimensional moduli space. There are of course a number of details to check in

Received July 9, 1998.

The author was partially supported by NSF Grant 4-50645. 
order to see that $I(f)$ does not depend on the various choices which were made; this is done below in section 2. The main issue is to see that the invariant is non-trivial, by computing it in suitable examples.

Our examples can be described rather simply, and reveal the 1-parameter invariant to be a sort of suspension of the ordinary YM or SW invariants. They arise from the observation that there are many pairs, say $X_{0}, X_{1}$ of nondiffeomorphic 4-manifolds which are homotopy equivalent, but which become diffeomorphic after connected sum with $\mathbb{C P}^{2}$. Let $Z^{\prime}$ denote this connected sum, and note that $Z^{\prime}$ contains two embedded spheres, say $S_{0}, S_{1}$, of selfintersection +1 , arising as complex lines in the two decompositions $X_{0} \# \mathbb{C P}^{2} \cong$ $Z^{\prime} \cong X_{1} \# \mathbb{C P}^{2}$. In typical examples, in fact, $Z^{\prime}$ decomposes completely into a connected sum of copies of $\mathbb{C P}^{2}$ and $\overline{\mathbb{C P}}^{2}$. Assuming this to be the case, a theorem of Wall [23] says that $Z^{\prime}$ supports enough diffeomorphisms so that we can assume that $S_{0}$ and $S_{1}$ are homologous; of course they are not isotopic.

Blow up two points on $Z^{\prime}$ to get $Z=Z^{\prime} \#_{2} \overline{\mathbb{C P}}^{2}$, which now contains, in addition to the $S_{i}$, two (-1)-spheres $E_{1}, E_{2}$ which are the the exceptional curves in $\overline{\mathbb{C P}}^{2}$. Associated to any sphere $\Sigma$ of self-intersection -1 in a 4 -manifold, there is a diffeomorphism $\rho^{\Sigma}$ inducing the 'reflection in $\Sigma$ ' on homology:

$$
\rho_{*}^{\Sigma}(\alpha)=\alpha+2(\Sigma \cdot \alpha) \Sigma .
$$

From the decomposition of $Z$ as $X_{0} \# \mathbb{C P}^{2} \#_{2} \overline{\mathbb{C P}}^{2}$, we have $(-1)$-spheres $S_{0}+$ $E_{1}+E_{2}$ and $S_{0}-E_{1}+E_{2}$, where the ' \pm ' signs refer to (oriented) connected sums of the spheres in $Z$. The composition of the reflection in these spheres is an orientation preserving diffeomorphism of $Z$ :

$$
f_{0}=\rho^{S_{0}+E_{1}+E_{2}} \circ \rho^{S_{0}-E_{1}+E_{2}} .
$$

Similarly, the decomposition $Z=X_{1} \# \mathbb{C P}^{2} \#_{2} \overline{\mathbb{C P}}^{2}$ gives rise to a diffeomorphism

$$
f_{1}=\rho^{S_{1}+E_{1}+E_{2}} \circ \rho^{S_{1}-E_{1}+E_{2}} .
$$

The fact that $S_{0}$ and $S_{1}$ are homologous implies that $f_{0}$ and $f_{1}$ are homotopic, so that $f=f_{1} \circ f_{0}^{-1}$ is homotopic to the identity of $Z$.

The main consequence of the work in this paper is that if there are suitable degree-0 Donaldson invariants $D_{X_{j}}$ with $D_{X_{0}} \neq D_{X_{1}}$, then the diffeomorphism $f$ is not smoothly isotopic to the identity. This follows from the basic properties of the invariants as outlined above, and the following calculations.

Theorem A. The 1-parameter Yang-Mills moduli space on the manifold $Z$ defines integer invariants $D_{Z}\left(f_{0}\right), D_{Z}\left(f_{1}\right)$ and $D_{Z}(f)$, which satisfy:

(1) $D_{Z}\left(f_{j}\right)=-4 D_{X_{j}}$ for $j=0,1$.

(2) $D_{Z}(f)=D_{Z}\left(f_{1}\right)-D_{Z}\left(f_{0}\right)$.

Corollary B. If $D_{X_{0}} \neq D_{X_{1}}$, then $f$ is not smoothly isotopic to the identity. 


\section{Definition of the invariants}

The precise nature of the invariants depends on which equations we are using, so we separate the discussion of the Seiberg-Witten and Yang-Mills equations. We will first describe a simple invariant, which corresponds to the degree-0 part of the Donaldson polynomial, and then indicate the modifications necessary to define Seiberg-Witten invariants of a diffeomorphism. The Seiberg-Witten invariant, in the version discussed in this paper, is not very useful, as we shall see. A more useful Seiberg-Witten invariant, as well as an extension of the Donaldson invariant to a polynomial invariant, will be described in subsequent papers $[21,20]$.

2.1. A simple invariant defined using the Yang-Mills equations. We begin by fixing the topological data and some of the notation. Let $Y$ be an oriented 4-manifold, with $b_{+}^{2}>1$, and suppose that an orientation of $H_{+}^{2}(Y)$ has been chosen. For simplicity, we will assume that $Y$ is simply-connected, although this is presumably not necessary. Fix an $\mathrm{SO}(3)$ bundle $P \rightarrow Y$, with non-trivial $w_{2}$, with the property that the formal dimension of the ASD moduli space $\mathcal{M}^{Y M}(P)$ is -1 . Note that this implies that $b_{+}^{2}(Y)$ is even, according to the usual dimension formula. Also, for a generic metric $g \in \operatorname{Met}(Y)$, the moduli space $\mathcal{M}^{Y M}(P ; g)$ is empty. (Here of course we use the non-triviality of $w_{2}$ and the condition that $b_{+}^{2}(Y)$ be positive to avoid reducibles. Also, unless there is some possible confusion with the Seiberg-Witten moduli space, we will drop the $Y M$ superscript.)

Although the moduli space is empty for generic $g$, one can consider instead a path of metrics $\left\{g_{t} \in \operatorname{Met}(Y) \mid t \in[0,1]\right\}$ and the '1-parameter moduli space'

$$
\tilde{\mathcal{M}}\left(P ;\left\{g_{t}\right\}\right)=\bigcup_{t}\left(\mathcal{M}\left(P ; g_{t}\right) \times\{t\}\right)
$$

which evidently has formal dimension 0 . (The topology on $\tilde{\mathcal{M}}$ is as a subspace of $\mathcal{A} / \mathcal{G} \times I$; we will usually omit the $t$-coordinate.) If $\left\{g_{t}\right\}$ is a generic path, then $\tilde{\mathcal{M}}$ will consist of a finite number of points, which we can count. Given an orientation on $H_{+}^{2}(X)$, we can assign orientations as well, and get an algebraic count of points, which we denote by $\# \tilde{\mathcal{M}}\left(P ;\left\{h_{t}\right\}\right)$.

Lemma 2.1. Suppose that $\mathcal{M}\left(\tilde{P} ; g_{t}\right)=\emptyset$ for $t=0,1$. Then for generic paths $\left\{g_{t}\right\} \subset$ Met and $\left\{g_{t}^{\prime}\right\} \subset$ Met with $g_{0}=g_{0}^{\prime}$ and $g_{1}=g_{1}^{\prime}$ we have that

$$
\# \tilde{\mathcal{M}}\left(P ;\left\{g_{t}\right\}\right)=\# \tilde{\mathcal{M}}\left(P ;\left\{g_{t}^{\prime}\right\}\right) \text {. }
$$

Proof. Since the space of metrics is contractible, one can find a 2-parameter family $g_{s, t} \in$ Met with $g_{0, t}=g_{t}$ and $g_{1, t}=g_{t}^{\prime}$. The hypothesis that $b_{+}^{2}>$ 2 implies that for a generic such family, there will be no reducible solutions to the ASD Yang-Mills equations defined using the metric $g_{s, t}$. Hence, for a 
generic family, the 2-parameter moduli space $\tilde{\tilde{\mathcal{M}}}$ will be a compact oriented 1dimensional cobordism with boundary $\tilde{\mathcal{M}}\left(P ;\left\{g_{t}^{\prime}\right\}\right)-\tilde{\mathcal{M}}\left(P ;\left\{g_{t}\right\}\right)$.

As a consequence, we have an integer invariant $D_{Y}\left(g_{0}, g_{1}\right)$ defined on pairs of generic metrics on $Y$; note that interchanging $g_{0}$ and $g_{1}$ reverses the orientation of the 1-parameter moduli space, and hence changes the sign of $D$. As in [6] a similar definition can be given of polynomial invariants defined using higherdimensional moduli spaces.

Suppose now that $f$ is an orientation-preserving diffeomorphism of $Y$, with the property that $f^{*} w_{2}(P)=w_{2}(P)$. Since $f$ has degree one, it automatically pulls back $p_{1}(P)$ to itself, and hence $f^{*}(P) \cong P$. It is worth remarking that while there is no canonical isomorphism between $f^{*}(P)$ and $P$, any two isomorphisms differ by composition with an element of the gauge group of $P$. Let $\alpha(f)$ be the spinor norm of $f$, i.e., $\alpha(f)= \pm 1$ depending on whether $f^{*}$ preserves or reverses orientation on $H_{+}^{2}$. Recall that an orientation of the moduli space is determined by the homology orientation, and the choice of an integral lift $c \in H^{2}(Y ; \mathbb{Z})$ for $w_{2}(P)$. Let $\beta(f)=(-1)^{\left(\frac{f^{*} c-c}{2}\right)^{2}}$, then it is readily checked that $\beta(f)$ does not depend on the choice of $c$. We will say that $f$ preserves or reverses orientation on the moduli space depending on whether the quantity $\alpha(f) \beta(f)= \pm 1$. (This terminology is explained by Corollary 3.28 of [5].)

Let $g_{0}$ be a metric for which the moduli space is empty; we propose to define an invariant $D_{Y}(f)=D_{Y}\left(g_{0}, f^{*} g_{0}\right)$, i.e., the count of points in a 1-parameter moduli space $\tilde{\mathcal{M}}\left(P ; g_{t}\right)$ where $g_{1}=f^{*} g_{0}$. Lemma 2.1 says that this is independent of the chosen path; a more subtle point is that the count is also independent of the initial metric $g_{0}$, so long as the final metric $g_{1}$ is $f^{*} g_{0}$.

Theorem 2.2. Suppose that $b_{+}^{2}(Y)>2$.Let $g_{0}$ and $k_{0}$ be generic metrics on $Y$, and let $g_{t}, k_{t} \in \operatorname{Met}(Y)$ be generic paths with $g_{1}=f^{*} g_{0}$ and $k_{1}=f^{*} k_{0}$. If $\alpha(f) \beta(f)=1$, then the algebraic number of points in the respective 1-parameter moduli spaces are the same:

$$
\# \tilde{\mathcal{M}}\left(P ;\left\{g_{t}\right\}\right)=\# \tilde{\mathcal{M}}\left(P ;\left\{k_{t}\right\}\right) .
$$

If $\alpha(f) \beta(f)=-1$, then the same statement holds modulo 2 .

Because of the theorem, we can unambiguously define

$$
D_{Y}(f)=\# \tilde{\mathcal{M}}\left(P ;\left\{g_{t}\right\}\right) \in \mathbb{Z} \text { or } \mathbb{Z}_{2},
$$

(depending on the sign of $\alpha(f) \beta(f)$,) using any generic $g_{0} \in \operatorname{Met}(Y)$.

Proof of Theorem 2.2. The key observation is that in general, for $g \in$ Met, there is an isomorphism of moduli spaces

$$
f^{*}: \mathcal{M}(P, g) \stackrel{\cong}{\rightrightarrows} \mathcal{M}\left(P, f^{*} g\right) .
$$

This means that $f^{*}$ is a homeomorphism, which is an isomorphism on the level of deformations, or more formally of locally ringed spaces. (As remarked above, 
$f^{*}$ is only well-defined after we have divided by gauge-equivalence, because it involves a choice of isomorphism between $f^{*} P$ and $P$.)

Start by choosing a generic path $K_{0, t}$ from $g_{0}=K_{0,0}$ to $k_{0}=K_{0,1}$, and note that $K_{1, t}=f^{*} K_{0, t}$ is thus necessarily generic. Now take arbitrary paths $g_{s}$ and $k_{s}$, and define $K_{s, 0}=g_{s}$ and $K_{s, 1}=k_{s}$. Thus we have a well-defined loop in the space of metrics, and as in the proof of Lemma 2.1, can fill it in with a generic 2-parameter family $K_{s, t}$.

Now consider the 2-parameter moduli space

$$
\tilde{\tilde{\mathcal{M}}}=\bigcup_{s, t} \mathcal{M}\left(P, K_{s, t}\right)
$$

which again is a compact 1-manifold with boundary $\partial \tilde{\tilde{\mathcal{M}}}$. (For the compactness, we need that there are no reducibles in a 2-parameter family, which is why we require that $b_{+}^{2}>2$.) By construction, $f^{*}$ induces an orientation preserving diffeomorphism between the part of the boundary lying on the path $K_{0, t}$ to the part lying on the path $K_{1, t}$. Put another way, the the algebraic number of points in the 1-parameter moduli space $\bigcup_{t} \mathcal{M}\left(P, K_{0, t}\right)$ must be the same as the number of points in $\bigcup_{t} \mathcal{M}\left(P, K_{1, t}\right)$. Hence the same must be true for the algebraic count of the points in $\bigcup_{s} \mathcal{M}\left(P, K_{s, 0}\right)$ and $\bigcup_{s} \mathcal{M}\left(P, K_{s, 1}\right)$. This proves the theorem; see figure 1 for an illustration.

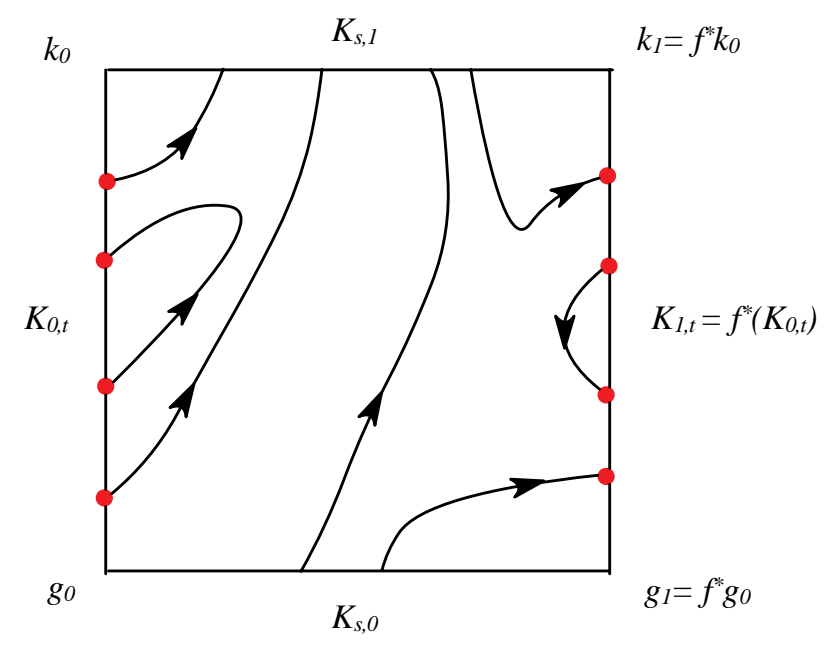

Figure 1

2.2. Invariants defined via the Seiberg-Witten equations. The definition of an invariant of diffeomorphisms based on the Seiberg-Witten equations is formally similar to that given above for the Yang-Mills equations. The main difference is that while the condition that $f$ preserves the isomorphism class of an $\mathrm{SO}(3)$ bundle is fairly weak, the condition that $f$ preserves a $\operatorname{Spin}^{\mathrm{c}}$ structure is much more restrictive. In this section, we discuss how to carry through the definition; those proofs which are essentially parallel to ones in section 2.1 are 
omitted or only sketched. Seiberg-Witten invariants of $f$ can be defined without the restriction that $f$ preserve a $\operatorname{Spin}^{\mathrm{c}}$ structure; this will be treated in more detail in [20].

Suppose that $(Y, g)$ is a Riemannian 4-manifold, with principal frame bundle $P=P(g) \rightarrow Y$. A $\operatorname{Spin}^{\mathrm{c}}$ structure on $Y$, from the point of view of principal bundles, is an $S^{1}$ bundle $\tilde{P} \rightarrow P$ which restricts on each fiber to the bundle $\operatorname{Spin}^{\mathrm{c}}(4) \rightarrow \mathrm{SO}(4)$. Although the $\mathrm{Spin}^{\mathrm{c}}$ structure is described in terms of the metric on $Y$, it is possible to show that a $\operatorname{Spin}^{\mathrm{c}}$ structure for one metric induces one for all metrics, as follows. Note that there is a 'universal' $\mathrm{SO}(4)$ bundle $\mathcal{P} \rightarrow Y \times \operatorname{Met}(Y)$ whose restriction to any slice $Y \times\{g\}$ is the frame bundle of $g$. Since the space $\operatorname{Met}(Y)$ is contractible, the bundle $\tilde{P} \rightarrow P(g)$ induces in a canonical way a bundle $\tilde{\mathcal{P}} \rightarrow Y \times$ Met which gives a $\operatorname{Spin}^{\mathrm{c}}$ structure for each metric. Under the projection $\Pi \rightarrow \operatorname{Met}(Y)$, the universal bundle pulls back to a bundle which we continue to denote $\tilde{\mathcal{P}} \rightarrow Y \times \Pi$. The spin representations give bundles $\mathcal{W}^{ \pm}$over $Y \times \Pi$ whose restrictions to $Y \times\{h\}$ are the ordinary spin bundles $W^{ \pm}(g)$.

Let $f$ be an orientation preserving diffeomorphism of $Y$, and note that if $\delta$ is a $g$ self-dual form, then $f^{*} \delta$ is $f^{*} g$ self-dual. Hence $f$ has a natural action on $\Pi$ :

$$
h=(g, \delta) \rightarrow f^{*} h=\left(f^{*} g, f^{*} \delta\right) .
$$

Associated to any Spin ${ }^{c}$ structure and $h \in \Pi$, we have the (perturbed) SeibergWitten equations for a connection $A$ on $\operatorname{det}\left(W^{+}\right)$and spinor $\phi \in \Gamma\left(W^{+}(g)\right)$

$$
\begin{aligned}
F_{A} & =q(\phi)+i \delta, \\
\not \partial_{A}(\phi) & =0 .
\end{aligned}
$$

Denote the solution space, modulo gauge equivalence, by $\mathcal{M}(\tilde{P} ; h)$ (or perhaps $\mathcal{M}^{S W}(\tilde{P} ; h)$ if it's not clear what equations are being discussed.) Let us assume that $b_{+}^{2}(Y)$ is even, and that for the $\operatorname{Spin}^{\mathrm{c}}$ structure $\tilde{P}$, the formal dimension of $\mathcal{M}^{S W}(\tilde{P})$ is -1 . Thus for generic $h \in \Pi$, the moduli space is empty. Once again, we consider the 1-parameter moduli space

$$
\tilde{\mathcal{M}}\left(\tilde{P} ;\left\{h_{t}\right\}\right)=\bigcup_{t} \mathcal{M}\left(\tilde{P}\left(h_{t}\right) ; h_{t}\right),
$$

which evidently has formal dimension 0 . Given an orientation on $H_{+}^{2}(Y)$, we can assign orientations to the points, and get an algebraic count of points, which we define to be $\operatorname{SW}\left(\tilde{P} ;\left\{h_{t}\right\}\right)$. We then have the analogue of Lemma 2.1, leading to an invariant of pairs of generic elements $h_{0}, h_{1} \in \Pi$.

Lemma 2.3. Suppose that $\mathcal{M}\left(\tilde{P} ; h_{t}\right)=\emptyset$ for $t=0,1$. Then for generic paths $\left\{h_{t}\right\} \subset \Pi$ and $\left\{h_{t}^{\prime}\right\} \subset \Pi$ with $h_{0}=h_{0}^{\prime}$ and $h_{1}=h_{1}^{\prime}$ we have that

$$
S W\left(\tilde{P} ;\left\{h_{t}\right\}\right)=S W\left(\tilde{P} ;\left\{h_{t}^{\prime}\right\}\right) .
$$


Now suppose that $f: Y \stackrel{\cong}{\rightrightarrows} Y$ is a diffeomorphism which preserves the orientation of $Y$ and acts on the orientation of $H_{+}^{2}(Y)$ with sign $\alpha(f)$, and that $\tilde{P}$ is a $\operatorname{Spin}^{\mathrm{c}}$ structure such that $f^{*}(\tilde{\mathcal{P}}) \cong \tilde{\mathcal{P}}$. Suppose that $h_{0} \in \Pi$ is generic, so that $\mathcal{M}\left(\tilde{P} ; h_{0}\right)$ is empty. Let $h_{1}=f^{*}\left(h_{0}\right)$, and take a generic path $\left\{h_{t}\right\}$ from $h_{0}$ to $h_{1}$. By analogy with Theorem 2.2, and its proof, we have:

Theorem 2.4. If $k_{0} \in \Pi$ is also a generic point, and $\left\{k_{t}\right\}$ is a generic path from $k_{0}$ to $k_{1}=f^{*} k_{0}$, then

$$
S W\left(\tilde{P} ;\left\{h_{t}\right\}\right)=S W\left(\tilde{P} ;\left\{k_{t}\right\}\right),
$$

as integers if $\alpha(f)=+1$ and modulo 2 if $\alpha(f)=-1$.

Because of the theorem, we can unambiguously define

$$
\mathrm{SW}(f, \tilde{P})=\mathrm{SW}\left(\tilde{P} ;\left\{h_{t}\right\}\right) \in \mathbb{Z} \text { or } \mathbb{Z}_{2},
$$

using any generic $h_{0} \in \Pi$.

The condition that $f$ preserve the $\operatorname{Spin}^{\mathrm{c}}$ structure can be relaxed somewhat. In fact, this condition was used only at one point in the proof, namely in identifying $\bigcup_{t} \mathcal{M}\left(\tilde{P}, K_{0, t}\right)$ with $\bigcup_{t} \mathcal{M}\left(\tilde{P}, K_{1, t}\right)$. Recall [15] that there is a natural involution $J$ on the space of $\mathrm{Spin}^{\mathrm{c}}$ structures, essentially given by replacing the spin bundle by its complex conjugate. The principal $\operatorname{Spin}^{\mathrm{c}}{ }_{4}$ bundle corresponding to a $\operatorname{Spin}^{\mathrm{c}}$ structure $\tilde{P}$ will be denoted $-\tilde{P}$. A fundamental observation is that $J$ behaves in a natural way with regard to the Seiberg-Witten equations, and in particular gives an isomorphism $J$ of moduli spaces

$$
\mathcal{M}^{S W}(\tilde{P} ; h) \cong \mathcal{M}^{S W}(-\tilde{P} ;-h) .
$$

Here, if $h=(g, \delta)$, then $-h$ will be the pair $(g,-\delta)$. The orientations on $\mathcal{M}^{S W}(\tilde{P} ; h)$ and $\mathcal{M}^{S W}(-\tilde{P} ;-h)$ differ $[15, \S 6.8]$ by a sign of $(-1)^{\epsilon}$, where $\epsilon=1+b_{1}(Y)+b_{+}^{2}(Y)+\operatorname{ind}_{C}(\not \partial)$. The assumption that $H_{1}(Y)=0$ and that the moduli space is -1 dimensional shows that in the case at hand, $\epsilon=\frac{b_{+}^{2}(Y)}{2}+1$. Let $\alpha(f)$ denote the spinor norm of $f$, i.e. $\alpha(f)= \pm 1$ depending on whether $f$ preserves or reverses the homology orientation.

Suppose that $f$ is an orientation preserving diffeomorphism, such that $f^{*} \tilde{P} \cong$ $-\tilde{P}$. Then we have the following variation of Theorem 2.4 .

Theorem 2.5. Let $f$ be an orientation preserving diffeomorphism, such that $f^{*} \tilde{P} \cong-\tilde{P}$. Let $h_{0}, k_{0}$ be generic points in $\Pi$, and suppose that $\left\{h_{t}\right\}$ is a generic path from $h_{0}$ to $h_{1}=f^{*} h_{0}$, with similarly chosen generic path $\left\{k_{t}\right\}$. Then

$$
S W\left(\tilde{P} ;\left\{h_{t}\right\}\right)=S W\left(\tilde{P} ;\left\{k_{t}\right\}\right),
$$

where the equality is over the integers if $(-1)^{\epsilon} \alpha(f)=1$ and is only modulo 2 if $(-1)^{\epsilon} \alpha(f)=-1$. 
The proof is essentially the same as that of Theorem 2.4, using the composition of diffeomorphisms

$$
\mathcal{M}(\tilde{P} ; h) \stackrel{f^{*}}{\rightarrow} \mathcal{M}\left(-\tilde{P}, f^{*} h\right) \stackrel{J}{\rightarrow} \mathcal{M}\left(\tilde{P},-f^{*} h\right) .
$$

To see that the assumption that $(-1)^{\epsilon} \alpha(f)=-1$ means that count of solutions is well-defined only modulo 2 , the reader should imagine reversing all the arrows on the curves in Figure 1 which meet the left-hand edge (where $t=0$.)

2.3. Some simple properties of the invariants. The invariants we have defined have many similarities with the ordinary Donaldson or Seiberg-Witten invariants; for instance they change sign when the homology orientation is reversed. In addition, they satisfy gluing properties analogous to those which hold for the ordinary invariants, the simplest of which we will exploit in section 3.1 to compute a non-trivial example. We will not develop this systematically, but instead record here some basic facts which will be needed in the computation. To avoid repeating essentially identical argument for the different sorts of invariants defined in the preceding sections, we will denote any of $D_{Y}(f)$, $D_{Y}\left(f, \Sigma_{1}, \ldots, \Sigma_{d}\right)$ or $\mathrm{SW}(f, \tilde{P})$ simply by $I(f)$. Similarly, $\Pi$ will denote either the space of metrics on $Y$ (if we are discussing Yang-Mills invariants) or the space of metrics and self-dual forms (for the case of Seiberg-Witten invariants), and $\mathcal{M}(h)$ will denote a moduli space. The first simple fact is that $I(f)$ is additive under composition. To put it in another way, the set of diffeomorphisms for which a given invariant is defined is a subgroup of $\operatorname{Diff}^{+}(Y)$, and $I$ gives a homomorphism to $\mathbb{Z}$ or $\mathbb{Z}_{2}$.

Lemma 2.6. Suppose that $f$ and $f^{\prime}$ are orientation preserving diffeomorphisms of $Y$, and that the invariants $I(f)$ and $I\left(f^{\prime}\right)$ are defined. Then

(1) $I\left(f^{\prime} \circ f\right)$ is defined, and equals $I\left(f^{\prime}\right)+I(f)$.

(2) $I\left(f^{-1}\right)$ is defined and equals $-I(f)$.

Proof. We make use of the fact that initial points $h_{0} \in \Pi$ and paths $\left\{h_{t}\right\} \in \Pi$ may be chosen arbitrarily. Let $h_{0}$ be a generic point in $\Pi$, and choose a generic path $h_{t}, t \in[0,1]$ with $h_{1}=f^{*} h_{0}$, so that $\# \mathcal{M}\left(\left\{h_{t}\right\}\right)=I(f)$. Choose a generic path, say $h_{t}^{\prime}, t \in[0,1]$, with $h_{0}^{\prime}=h_{1}$, so that $\# \mathcal{M}\left(\left\{h_{t}^{\prime}\right\}\right)=I\left(f^{\prime}\right)$. Putting the two together gives a path $h * h^{\prime}$, which calculates $I\left(f^{\prime} \circ f\right)$. But manifestly the (algebraic) number of points in the corresponding 1-parameter moduli space is $\# \mathcal{M}\left(\left\{h_{t}\right\}\right)+\# \mathcal{M}\left(\left\{h_{t}^{\prime}\right\}\right)=I\left(f^{\prime}\right)+I(f)$.

The second part follows by considering the initial point in $\Pi$ for $f^{-1}$ to be $f^{*} h_{0}$, and using the path $h_{1-t}$. The reversal in the path changes the orientation of the 1-parameter moduli space, which accounts for the minus sign.

The other important principle to note is that $I(f)$ depends only on the isotopy class of $f$. 
Lemma 2.7. Suppose that $f_{0}$ and $f_{1}$ are smoothly isotopic diffeomorphisms of a 4-manifold $Y$, and that one of the invariants $I(f)$ is defined. Then $I\left(f_{0}\right)=I\left(f_{1}\right)$.

Proof. Note that if $f_{0}$ is isotopic to $f_{1}$, then $f_{1} \circ f_{0}^{-1}$ is isotopic to the identity. So by the previous lemma, and the obvious remark that the identity has trivial invariant, it suffices to show that $I\left(f_{1}\right)=0$ if $f_{1}$ is isotopic to the identity. Let $f_{t}$ be an isotopy from the identity to $f_{1}$. Choose $h_{0} \in \Pi$ for which the moduli space $\mathcal{M}\left(Y ; h_{0}\right)$ is empty, and consider the path $h_{t}=f_{t}^{*} h_{0}$. As remarked in the proof of Theorem 2.2, for each $t \in[0,1]$ the moduli space $\mathcal{M}\left(Y ; h_{t}\right)$ is isomorphic to $\mathcal{M}\left(P ; h_{0}\right)$, i.e., is empty. Hence $I\left(f_{1}\right)=0$.

\section{Smoothly non-isotopic diffeomorphisms}

In this section, we give the detailed construction of the diffeomorphism described in the introduction, and use the invariant $D(f)$ to show that it is not smoothly isotopic to the identity. As described in the introduction, the diffeomorphism is a composition of four 'reflections' on a manifold $Z$, where each reflection is associated to a certain 2 -sphere embedded in $Z$. So as an initial step, we discuss the 1-parameter gauge theory associated to a single reflection.

The manifold $Z$ will be written as $X_{0} \# \mathbb{C P}^{2} \#_{2} \overline{\mathbb{C P}}^{2}$, with some topological hypotheses on $X_{0}$ needed for the calculation to succeed. These depend on whether one wants to calculate $D(f)$ or $\mathrm{SW}(f)$, and somewhat surprisingly are considerably more restrictive in the Seiberg-Witten case. In either case, we assume that $H_{1}\left(X_{0}\right)=0$, and that $b_{+}^{2}$ is odd and greater than 1 . In addition, for the Yang-Mills version, we require that:

$\begin{array}{ll}\left(*_{Y M}\right) & \text { There exists an } \mathrm{SO}(3) \text { bundle } P_{0} \rightarrow X_{0} \\ w_{2}\left(P_{0}\right) \neq 0 \text { and } \operatorname{dim}\left(\mathcal{M}^{Y M}\left(P_{0}\right)\right)=0 .\end{array}$

The requirement for the Seiberg-Witten version is discussed in section 3.3.

Let $N=\mathbb{C P}^{2} \#_{2} \overline{\mathbb{C P}}^{2}$, and let $S, E_{1}, E_{2}$ be the obvious embedded 2-spheres of self-intersection \pm 1 , and let $\Sigma_{ \pm}=S \pm E_{1}+E_{2}$ be spheres of square -1 . For $\Sigma=\Sigma_{+}$or $\Sigma_{-}$, there is a diffeomorphism $\rho^{\Sigma}$ of $N$, inducing in homology a reflection in $\Sigma$, in other words, $\rho_{*}^{\Sigma}(x)=x+2(x \cdot \Sigma) \Sigma$, where '.' is the intersection product. (In cohomology, $\left(\rho^{\Sigma}\right)^{*}$ is given by the same formula, where $\Sigma$ is replaced by $\sigma=P D(\Sigma)$ and is replaced by $\cup$.) In particular, letting $s, e_{1}, e_{2}$ be the Poincaré duals of $S, E_{1}, E_{2}$, we have $\left(\rho^{\Sigma}\right)^{*}(s)=s+2(s \cup \sigma) \sigma=3 s+2\left(e_{2} \pm e_{1}\right)$. Hence $\rho^{\Sigma}$ is orientation preserving on $H_{+}^{2}(N)$. Choose the orientation in which $s$ is in the positive cone.

Fix a point $p$ in $N$, and choose specific diffeomorphisms $\rho^{\Sigma}$ which are equal to the identity in some fixed neighborhood of $p$. For any 4-manifold $X_{0}$, such a diffeomorphism glues together with the identity map of $X_{0}$ to give a diffeomorphism $f^{\Sigma}$ on the connected sum $Z=X_{0} \# N$. Let $L(=L(1,1,1))$ be the complex line bundle over $N$ with first Chern class $s+e_{1}+e_{2}$. The bundle $P_{0}$ 
may be glued together with $L \oplus \mathbb{R}$ to give an $\mathrm{SO}(3)$ bundle $P_{Z} \rightarrow Z$, which will be the basis for our computation.

Any single diffeomorphism $f^{\Sigma}$ reverses the orientation of the moduli space, and so has only $\mathbb{Z}_{2}$ invariants. In fact, it turns out (see the proof of Theorem 3.1 and the discussion after Theorem 3.4) that all of these $\mathbb{Z}_{2}$ invariants are trivial. To get a more interesting computation, we compose two reflections, to get $f_{0}=$

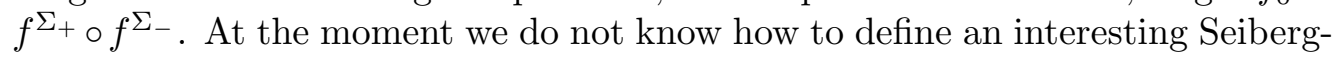
Witten invariant of $f_{0}$, so we will concentrate mainly on the calculation of a Donaldson invariant $D_{Z}\left(f_{0}\right)$. In the next section, we will verify the first part of Theorem A of the introduction.

Theorem 3.1. Suppose that $P_{0} \rightarrow X_{0}$ is an $\mathrm{SO}(3)$ bundle with non-trivial $w_{2}$, so that the 0-dimensional Donaldson invariant $D_{X_{0}}$ is defined. Then the invariant $D_{Z}\left(f_{0}\right) \in \mathbb{Z}$ is defined, and $D_{Z}\left(f_{0}\right)=-4 D_{X_{0}}$.

It is worth noting that this theorem depends only on the homotopy type of $N$, and on the maps induced in cohomology by the restriction of $f_{0}$ to $N$.

3.1. Wall-crossing and the 1-parameter invariant. The calculation of the invariant $D_{Z}\left(f_{0}\right)$ depends on a simple wall-crossing argument in the style of [4, 10]. We give the details of this argument in this subsection. First we need to review the chamber structure associated to reducible connections on a particular $\mathrm{SO}(3)$ bundle over $N$.

On $N$, consider the $\mathrm{SO}(3)$ bundle $P$ with associated $\mathbb{R}^{3}$ bundle $L \oplus \mathbb{R}$, where $L$ is the complex line bundle with $c_{1}=s+e_{1}+e_{2}$. Reductions of $P$ to an $\mathrm{SO}(2)$ bundle are in one-to-one correspondence with cohomology classes $(a, b, c) \in$ $H^{2}(N ; \mathbb{Z})$ where $c$ is positive, $a, b$, and $c$ are odd and satisfy $c^{2}-a^{2}-b^{2}=-1$. For any metric $g$ on $N$, there is a unique harmonic form $\omega_{g} \in \Omega_{+}^{2}(N)$ with

$$
\int_{N} \omega_{g} \wedge \omega_{g}=1 \text { and }\left[\omega_{g}\right] \cup s>0 .
$$

Denote by $\mathcal{H} \subset H^{2}(N ; \mathbb{R})$ the set of cohomology classes satisfying (1). The line bundle corresponding to a triple $(a, b, c)$ admits a $g$-ASD connection if and only if $\omega_{g}$, as an element of $H^{2}(N ; \mathbb{R})$, lies on the 'wall'

$$
W(a, b, c)=\{(x, y, z) \in \mathcal{H} \mid c z-a x-b y=0\} .
$$

It is readily verified that distinct walls are in fact disjoint (cf. [9, Chapter II]; a picture of the walls may be found below in Figure 2.) The walls are transversally oriented, using the following convention. Let $\epsilon(a, b, c)$ be the sign with which the orientation of $\mathcal{M}\left(P_{N}\right)$ induced by the reduction to $L(a, b, c)$ compares to the given one, i.e. that induced by the reduction to $L(1,1,1)$. According to [5], this is given by

$$
\epsilon(a, b, c)=(-1)^{\left[\left(\frac{c-1}{2}\right)^{2}-\left(\frac{a-1}{2}\right)^{2}-\left(\frac{b-1}{2}\right)^{2}\right]}
$$

The transverse orientation of $W(a, b, c)$ is chosen so that the positive side consists of those $(x, y, z)$ with $\epsilon(a, b, c)(c z-a x-b y)>0$. Let us write $\mathcal{W}$ for the union 
of the $W(a, b, c)$; it is a transversally oriented, locally finite, proper submanifold of $\mathcal{H}$.

Write $Z=X \# N$; eventually $X$ will be either $X_{0}$ or $X_{1}$. We assume that we have an $\mathrm{SO}(3)$-bundle $P_{X} \rightarrow X$ with non-trivial $w_{2}$, for which the moduli space is formally 0-dimensional. Consider a metric $g^{X}$ which is Euclidean in a neighborhood of the point $p$ where the connected sum is made. The complement of $p$ has a neighborhood which is conformally the cylinder $S^{3} \times[-T, 1)$. By a small perturbation away from this tube, it may be assumed that the moduli space $\mathcal{M}^{Y M}\left(P_{X} ; g^{X}\right)$ is smooth; the fact that $w_{2} \neq 0$ readily implies that it is also compact. Choosing an orientation of $H_{+}^{2}(X)$, together with an integral lift $c_{X}$ of $w_{2}\left(P_{X}\right)$ yields the Donaldson invariant $D_{X}$.

The same data gives a homology orientation for $Z$; it is the homology orientation $X$, followed by the choice of generator $s \in H^{2}\left(\mathbb{C P}^{2}\right) \cong H_{+}^{2}(N)$. We fix the integral lift $c_{X} \oplus\left(s+e_{1}+e_{2}\right) \in H^{2}(X) \oplus H^{2}(N)$ for $w_{2}\left(P_{Z}\right)$. Note that

$$
f^{*}\left(c_{X} \oplus\left(s+e_{1}+e_{2}\right)\right)=c_{X} \oplus\left(-3 s+e_{1}-3 e_{2}\right),
$$

so that the data needed to define the integral invariant $D_{Z}\left(f_{0}\right)$ is present.

Choose a metric $g_{0}^{N}$ on $N$ with a similar structure near the connected sum point, and glue together to get a metric $g_{0}$ on $Z$, so that $Z$ contains a long tube (of length $2 T)$. By a perturbation of $g_{0}^{N}$, supported away from $p$, we can assume that the harmonic form $\omega_{g_{0}^{N}}$ (or $\omega_{0}$ for short) does not lie on any wall. Furthermore, since the dimension of $\mathcal{M}^{Y M}\left(P_{N}\right)$ is $-2 p_{1}(L \oplus \mathbb{R})-3\left(b_{+}^{2}(N)+1\right)=-4$, we can assume that

$$
\mathcal{M}^{Y M}\left(P_{N} ; g_{0}^{N}\right)=\emptyset .
$$

Suppose that $g_{1}^{N}$ is a second metric which satisfies the same genericity properties and has the same behavior near $p$; of course $g_{1}^{N}$ defines a self-dual harmonic form $\omega_{1}$. This metric may also be glued to the metric $g^{X}$ to obtain a metric $g_{1}$ on $Z$. We want to evaluate the 1-parameter Donaldson invariant $D_{Z}\left(g_{0}, g_{1}\right)$, at least in the limit when the tube length $T$ is large. To this end, choose any path in $\gamma \subset \mathcal{H}$ from $\omega_{0}$ to $\omega_{1}$ which is transverse to all of the walls.

Lemma 3.2. If $T$ is sufficiently large, then $D_{Z}\left(g_{0}, g_{1}\right)=2(\gamma \cdot \mathcal{W}) D_{X}$.

Proof. We begin by remarking that the intersection number in the statement of the lemma is indeed well-defined, i.e., independent of the path in $\mathcal{H}$ provided the endpoints are fixed. Thus, we are free to choose any path of metrics on $Z$; our path will be of the form $g^{X} \# g_{t}^{N}$, where the metrics on $N$ are chosen as follows. Make an initial choice of path so that the associated path of harmonic forms is transverse to $\mathcal{W}$. Consider a compact subinterval $J$ of $[0,1]$ for which the associated harmonic forms do not lie on $\mathcal{W}$. Because the parameterized moduli space $\mathcal{M}^{Y M}\left(P_{N}, g_{t}^{N}\right)$ is formally of dimension -3 , a small perturbation (relative to the endpoints of $J$ ) will ensure that $\mathcal{M}^{Y M}(N ; J)=\emptyset$. This fails to hold for intervals whose associated path of harmonic forms meet $\mathcal{W}$, because one cannot perturb away the reducible connections. 
Each intersection point of $\gamma$ and $\mathcal{W}$ thus contributes to $D_{Z}\left(g_{0}, g_{1}\right)$, and we need to show that the contribution is given by $\pm 2 D_{X}$ when the intersection is transverse, and to evaluate the sign. Consider, for $t \in(-\epsilon, \epsilon)$, a path of metrics so that the associated path of harmonic forms crosses the wall $W(a, b, c)$ transversally at $t=0$. For each $t$, there is a unique unitary connection $A_{t}^{N}$ on $L$ such that $\frac{1}{2 \pi i} F_{A_{t}}$ is $g_{t}^{N}$-harmonic. The assumption that the path $\omega_{G_{t}^{N}}$ crosses the wall transversally at $t=0$ means precisely that $A_{0}^{N}$ is anti-self-dual, and that the function

$$
h(t)=\left(a e_{1}+b e_{2}+c s\right) \cup \omega_{g_{t}^{N}}=\frac{1}{2 \pi i} F_{A_{t}} \cup \omega_{g_{t}^{N}}=\frac{1}{2 \pi i} F_{A_{t}}^{+} \cup \omega_{g_{t}^{N}},
$$

has non-zero derivative at 0 .

One can glue the whole path $A_{t}^{N}$ to any given ASD connection $A^{X}$ on $P_{X}$, and consider the problem of deforming the resulting path to ASD connections on $P_{Z}$. The only possible solutions (for sufficiently large tube length) are when $t$ is near zero, and may be described in terms of the Kuranishi picture for the 1-parameter gluing problem. The Kuranishi picture (compare [3, 12]) for the gluing problem is an $\mathrm{SO}(2)=\operatorname{Stab}\left(A_{0}^{N}\right)$-equivariant map

$$
\psi:(-\epsilon, \epsilon) \times \mathcal{H}^{1}\left(A_{0}^{N}\right) \times \mathcal{H}^{1}\left(A^{X}\right) \times \mathrm{SO}(3) \rightarrow \mathcal{H}_{+}^{2}\left(A_{0}^{N}\right) \times \mathcal{H}_{+}^{2}\left(A^{X}\right),
$$

so that the part of the moduli space $\mathcal{M}\left(P_{Z} ;\left\{g_{t}\right\}\right)$ for $t \in(-\epsilon, \epsilon)$ is modeled by $\psi^{-1}(0) / \mathrm{SO}(2)$.

Since $A^{X}$ is a smooth point in the 0-dimensional space $\mathcal{M}^{Y M}\left(P_{X}\right)$, it has vanishing cohomology groups $\mathcal{H}^{1}\left(A^{X}\right)$ and $\mathcal{H}_{+}^{2}\left(A^{X}\right)$. On the $N$ side, the cohomology groups split as $\mathcal{H}^{*}(L) \oplus \mathcal{H}^{*}(N ; \mathbb{R})$, where the first summand gets a complex structure from $L$. The index calculation shows that $\mathcal{H}^{1}\left(A^{N}\right) \cong \mathcal{H}^{1}(L) \cong \mathbb{C}^{r}$ and $\mathcal{H}_{+}^{2}\left(A^{N}\right) \cong \mathcal{H}_{+}^{2}(L) \oplus \mathcal{H}_{+}^{2}(N ; \mathbb{R}) \cong \mathbb{C}^{r+1} \oplus \mathbb{R}$ for some $r \geq 0$. In fact [7] by a small perturbation of the whole path of metrics on $N$, one can arrange that $r=0$, which we do for convenience. Hence $\psi$ is an $\mathrm{SO}(2)$ equivariant map

$$
(-\epsilon, \epsilon) \times \mathrm{SO}(3) \rightarrow \mathbb{C} \times \mathbb{R}
$$

The projection of $\psi$ onto the last component (ie the one corresponding to $\left.\mathcal{H}_{+}^{2}(N ; \mathbb{R})\right)$ is given, at the point $(0,0)$ by the map $h$ described in equation $(2)$, up to first order. Thus the local contribution of $A^{X}$ is given by counting the zeros of an $\mathrm{SO}(2)$ equivariant map

$$
\psi_{0}: \mathrm{SO}(3) \rightarrow \mathbb{C}
$$

Such a map is equivalent to a section of the complex line bundle

$$
\mathrm{SO}(3) \times_{\mathrm{SO}(2)} \mathbb{C} \rightarrow \mathrm{SO}(3) / \mathrm{SO}(2) \cong S^{2},
$$

which is readily seen to be the tangent bundle of $S^{2}$. Since the Euler class of the tangent bundle is 2 , each point $A^{X} \in \mathcal{M}\left(P_{X}\right)$ contributes \pm 2 to the 1 parameter invariant. To compute the sign, note first that the determinant bundle for $d^{*}+d^{+}$has an orientation coming from the reduction into $L(a, b, c) \oplus \mathbb{R}$. 
As remarked before, this differs from the orientation arising from the original splitting into $L(1,1,1) \oplus \mathbb{R}$, with sign given by $\epsilon(a, b, c)$. Hence each intersection point contributes $2 \epsilon$ times the sign of $h^{\prime}(0)$, which is exactly the intersection number with $W(a, b, c)$, using the prescribed transverse orientation.

With this basic step in hand, we can compute the invariant for the diffeomorphism $f_{0}$ described above.

Proof of Theorem 3.1. Observe that each of the diffeomorphisms $f^{\Sigma_{ \pm}}$is orientation preserving, so that to see that there is a well-defined invariant $D_{Z}\left(f_{0}\right)$, it suffices to check the effect of $f_{0}$ on $c_{1}(L)=s+e_{1}+e_{2}$. It is readily verified that $f_{0}^{*}\left(s+e_{1}+e_{2}\right)=5 s+e_{1}+5 e_{2}$, which shows that $f_{0}$ preserves $P_{0}$, and also that the action on the moduli space is orientation preserving. Hence the integer invariant $D_{Z}\left(f_{0}\right)$ is defined. According to the calculation in Lemma 3.2, we only have to check, for any $\omega_{0} \in \mathcal{H}$, the intersection number of a path in $\mathcal{H}$ from $\omega_{0}$ to $f_{0}^{*}\left(\omega_{0}\right)$ with the walls $\mathcal{W}$. But this is easily seen to be be -2 , by taking a point such as $(0,0,1)$; see the following figure and discussion.

A few of the walls are illustrated below, in the upper half-space model of $\mathcal{H}$. The point $(0,0)$ (which corresponds to $(0,0,1)$ in the hyperboloid $z^{2}-x^{2}-y^{2}=1$ ) is indicated with a small + sign, together with its image under $f_{0}^{*}$. The path from $(0,0)$ to its image crosses the walls corresponding to the reductions $L(1,1,1)$ and $L(1,3,3)$, each with a negative orientation. As a check of the basic set-up, a second point $(-1 / 2,-1 / 2)$, as is its image, is indicated by a small circle $\circ$. The path from $(-1 / 2,-1 / 2)$ to $f_{0}^{*}(-1 / 2,-1 / 2)$ crosses the walls $W(-1,-1,1)$, $W(1,1,1), W(1,3,3)$, and $W(7,11,13)$. The first crossing counts for +2 , while the others count -2 , for a net of -4 .

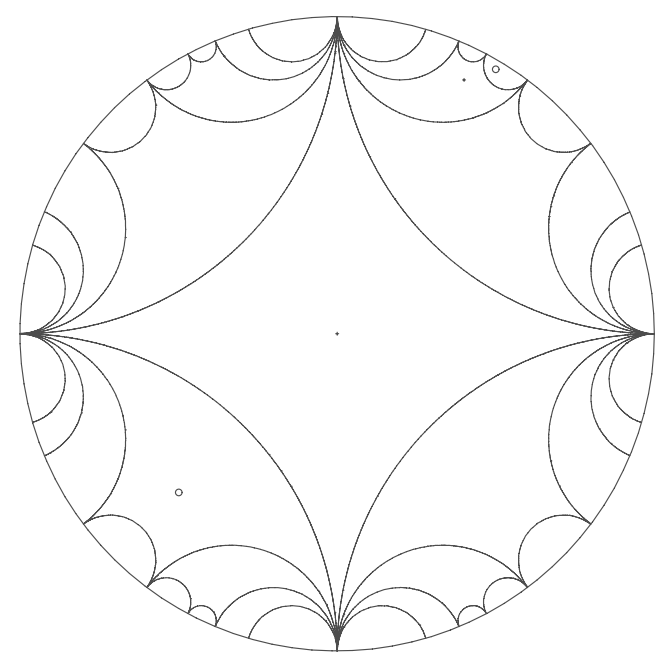

Figure 2

3.2. Non-isotopic diffeomorphisms. As described in the introduction, there are many examples of pairs of manifolds $X_{0}, X_{1}$ with $X_{0} \# \mathbb{C P}^{2} \cong X_{1} \# \mathbb{C P}^{2}$, 
where the diffeomorphism may be assumed to carry the homology class of the (+1)-sphere $S_{0}$ to that of $S_{1}$. Adding two copies of $\overline{\mathbb{C P}}^{2}$ gives $Z$, supporting two homotopic diffeomorphisms $f_{0}, f_{1}$, which are precisely of the type discussed above. Let $f=f_{1} \circ f_{0}^{-1}$.

Theorem 3.3. $D_{Z}(f)$ is a well-defined integer invariant and is given by

$$
D_{Z}(f)=D_{Z}\left(f_{1}\right)-D_{Z}\left(f_{0}\right)=-4\left(D_{X_{1}}-D_{X_{0}}\right) .
$$

In particular, if $D_{X_{0}} \neq D_{X_{1}}$, then $f$ is a diffeomorphism which is topologically isotopic to the identity, but not smoothly so.

Proof. Because $S_{0}$ and $S_{1}$ are homologous, $f_{0}$ and $f_{1}$ induce the same map on homology, and hence $f$ induces the identity map on homology. It follows $[2,19]$ that $f$ is homotopic and in fact topologically isotopic to the identity of $Z$. Since $f$ induces the identity on $H_{2}(Z)$, it follows that $D_{Z}(f) \in \mathbb{Z}$ is defined. Lemma 2.6, together with the computation in Lemma 3.2, gives the calculation of $D_{Z}(f)$. If $D_{X_{0}}$ and $D_{X_{1}}$ are different, then it follows from Lemma 2.7 that $f$ is not isotopic to the identity.

3.3. The Seiberg-Witten version. It seems worth asking whether invariants based on the Seiberg-Witten equations can be used to detect the non-triviality of a diffeomorphism. Because the diffeomorphism $f^{\Sigma^{+}} \circ f^{\Sigma^{-}}$acts non-trivially on the set of Spin ${ }^{\mathrm{c}}$ structures, even up to the action of $J$, the current methods do not give rise to a useful invariant. However, using the ideas discussed in section 2.2 we can in some circumstances define a mod 2 invariant of a diffeomorphism built from a single reflection, for example $f^{\Sigma^{+}}$. (Of course the hope would be to get different answers by taking $X$ to be the manifolds $X_{0}$ and $X_{1}$.) In order to do this, we need to assume that the manifold $X$ has a Spin ${ }^{\mathrm{c}}$-structure $\tilde{P}_{X} \rightarrow X$ with $c_{1}\left(\tilde{P}_{X}\right)=0$ and $\operatorname{dim}\left(\mathcal{M}^{S W}\left(\tilde{P}_{X}\right)\right)=0$. This hypothesis implies that $X$ is a spin manifold; using Rohlin's theorem, and the formula for the dimension of $\mathcal{M}^{S W}\left(\tilde{P}_{X}\right)$, one readily deduces that $b_{+}^{2}(X) \equiv-1(\bmod 4)$.

Let $\tilde{P}_{Z}$ be the Spin ${ }^{\text {c}}$-structure on $Z$ which is a connected sum of $\tilde{P}_{X}$ with

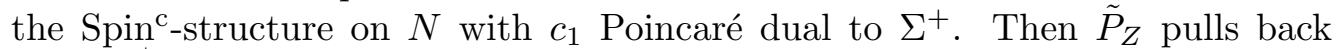
(via $f_{0}^{\Sigma^{+}}$) to $J\left(\tilde{P}_{Z}\right)$, and so by Theorem 2.5 there is an invariant $\operatorname{SW}\left(f, \tilde{P}_{Z}\right)$. Since $b_{+}^{2}(Z) \equiv 0(\bmod 4)$, the quantity $\epsilon$ appearing in that theorem is 1 , and so $\mathrm{SW}\left(f, \tilde{P}_{Z}\right)$ is only defined modulo 2 . In any event, we can still calculate $\mathrm{SW}\left(f, \tilde{P}_{Z}\right)$ by the wall-crossing technique. The details of the Kuranishi picture as the wall is crossed are slightly different and may be deduced from the calculation in [12]. The result (whose proof we omit) should be compared with Theorem 3.1.

Theorem 3.4. Let $\tilde{P}_{X} \rightarrow X$ be a Spin ${ }^{\mathrm{c}}$-structure satisfying $c_{1}\left(\tilde{P}_{X}\right)=0$ and $\operatorname{dim}\left(\mathcal{M}^{S W}\left(\tilde{P}_{X}\right)\right)=0$. Then the invariant $S W\left(f, \tilde{P}_{Z}\right)$ described above is equal (modulo 2) to $S W\left(\tilde{P}_{X}\right)$. 
The rather unfortunate punchline to this story is that the mod 2 invariant $\mathrm{SW}\left(f, \tilde{P}_{Z}\right)$ does not depend on $X$ in any interesting way. This follows from a theorem of Morgan and Szabó [16] which states that the invariant $\operatorname{SW}\left(\tilde{P}_{X}\right)$ for the sort of $X$ we considered is determined $(\bmod 2)$ simply by $b_{+}^{2}$. In fact, if $b_{+}^{2}(X)=3$, then the Seiberg-Witten invariant is odd, and if $b_{+}^{2}(X)>3$, then the Seiberg-Witten invariant is even. So as it stands, we do not know how to use a Seiberg-Witten invariant to detect non-isotopic diffeomorphisms.

3.4. Discussion. The construction of the examples seems in some respects more complicated than necessary. Before the addition of the two copies of $\overline{\mathbb{C P}}^{2}$, we had the manifold $Z^{\prime}$, containing two spheres $S_{0}, S_{1}$ of self-intersection +1 . Associated to each of these is a diffeomorphism $f_{i}^{\prime}$, where the induced maps in homology now satisfy $\left(f_{i}\right)_{*}(x)=x-2\left(x \cdot S_{i}\right) S_{i}$. It is natural to conjecture that these diffeomorphisms are not isotopic (as before, they could certainly be arranged to be homotopic) and indeed that was our original conception. However, the construction given in this paper does not seem to provide invariants of the $f_{i}^{\prime}$ which would encode the difference in Donaldson (or Seiberg-Witten) invariants of the manifolds $X_{i}$.

One aspect of this is related to the other complication in the examples, namely that the diffeomorphisms $f_{0}$ and $f_{1}$ are not reflections, but are instead compositions of such. The calculation of the action on orientation of the moduli space shows that a single reflection reverses orientations, and so leads only to a $\mathbb{Z}_{2}$ invariant, which we have seen does not contain much useful information about isotopy of diffeomorphisms. This calculational fact has a more conceptual basis, which can be seen if one interprets our invariants as giving rise to cocycles of some sort in $H^{1}\left(\operatorname{Met}^{*}(Z) / \operatorname{Diff}^{+}(Z)\right)$. A diffeomorphism of finite order preserves a metric on $Z$, and hence the action on $\operatorname{Met}(Z)$ has fixed points. One gets a better quotient space considering $\operatorname{Met}^{*}(Z)$, which consists of metrics with trivial isometry group. One would expect that if $f$ had order $n$, then the count of solutions around a loop in $\operatorname{Met}^{*}(Z)$ linking the fixed point would be well-defined only modulo $n$. If one views the examples in this light, then it is clear that one should only have a mod 2 invariant associated to a single reflection. The composition of two reflections (as used in the examples) has infinite order, circumventing this difficulty.

An interpretation of our invariants as cohomology classes is the proper setting for the generalization to invariants defined by families of dimension greater than one. We plan to return to this in a future paper.

\section{Acknowledgement}

I'd like to thank Tom Mrowka for listening to an earlier, more complicated version of this work, and for asking the right questions. 


\section{References}

1. J. Bryan and Leung, N.C., The enumerative geometry of K3 surfaces and modular forms. Preprint, available from http://xxx.lanl.gov/e-print/alg-geom/9711031, 1997.

2. T. Cochran and N. Habegger, On the homotopy theory of simply connected four manifolds, Topology 29 (1990), 419-440.

3. S. Donaldson and P. Kronheimer, The Geometry of Four-Manifolds, Clarendon Press, Oxford, 1990.

4. S.K. Donaldson, Irrationality and the h-cobordism conjecture, J. Diff. Geo. 26 (1987), 141-168.

5. - The orientation of Yang-Mills moduli spaces and 4-manifold topology, J. Diff. Geo. 26 (1987), 397-428.

6. — , Yang-Mills invariants of four-manifolds, in Geometry of low-dimensional manifolds, 1 (Durham, 1989), vol. 150 of London Math. Soc. Lecture Note Ser., Cambridge Univ. Press, Cambridge, 1990, 5-40.

7. D. Freed and K. Uhlenbeck, Instantons and 4-Manifolds, MSRI Lecture notes, SpringerVerlag, 1984.

8. M. Freedman and F. Quinn, Topology of 4-manifolds, Princeton University Press, Princeton, N.J., 1990.

9. R. Friedman and J. Morgan, On the diffeomorphism types of certain complex surfaces: I, J. Diff. Geo. 27 (1988), 297-369.

10. D. Kotschick, $S O(3)$-invariants for 4-manifolds with $b_{2}^{+}=1$, Proc. Lond. Math. Soc. 63 (1991), 426-448.

11. P. Kronheimer, Some non-trivial families of symplectic structures. Preprint, available from http://www.math.harvard.edu/ kronheim/diffsymp.pdf, 1997.

12. P. Kronheimer and T. Mrowka, The genus of embedded surfaces in the projective plane, Math. Res. Lett. 1 (1994), 797-808.

13. S. Kwasik, Low-dimensional concordances, Whitney towers and isotopies, Math. Proc. Camb. Phil. Soc. 102 (1987), 103-119.

14. T.-J. Li and A.-K. Liu, Family Sieberg-Witten invariant, preprint, 1998.

15. J.W. Morgan, The Seiberg-Witten equations and applications to the topology of smooth four-manifolds, vol. 44 of Mathematical Notes, Princeton University Press, Princeton, NJ, 1996.

16. J.W. Morgan and Z. Szabó, Homotopy K3 surfaces and mod 2 Seiberg-Witten invariants, Math. Res. Lett. 4 (1997), 17-21.

17. B. Perron, Pseudo-isotopies et isotopies en dimension 4 dans la catégorie topologique, C. R. Acad. Sci. Paris 299 (1984), 455-458.

18. B. Perron, Pseudo-isotopies et isotopies en dimension quatre dans la catégorie topologique, Topology 25 (1986), 381-397.

19. F. Quinn, Isotopy of 4-manifolds, J. Diff. Geo. 24 (1986), 343-372.

20. D. Ruberman, Isotopy and the Seiberg-Witten equations. In preparation.

21. - A polynomial invariant of diffeomorphisms of 4-manifolds. Preprint (1998).

22. P. Seidel, $\pi_{1}$ of symplectic automorphism groups and invertibles in quantum homology rings, Geom. Funct. Anal. 7 (1997), 1046-1095.

23. C.T.C. Wall, Diffeomorphisms of 4-manifolds, Jour. Lond. Math. Soc. 39 (1964), 131-140.

Department of Mathematics,Brandeis University,Waltham, MA 02254

E-mail address: ruberman@binah.cc.brandeis.edu 